\title{
Analysis on the Development and Innovation of Oil Recovery Engineering Techniques in the New Period
}

\author{
Shi hong $\mathrm{Li}^{1 *}$ \\ M aozhou operation area, No.1 oil production plant, Huabei Oilfield, Renqiu City, Hebei Province, Renqiu, Hebei, 062552, China.
}

\begin{abstract}
Since the new period of reform and opening up in 1978, China has witnessed rapid social and economic development, higher living standards, and greater petroleum demands, which further promotes the scale and intensity of oil recovery. Oil recovery is progressing with greater difficulties. Traditional oil recovery techniques can no longer meet the demands for petroleum against the backdrop of today's national economic development, and so we need to further enhance the efficiency and quality of oil recovery. This paper analyzes the research status of oil recovery techniques at home and abroad, illustrates the basic principles of the techniques and the development difficulties, and then explores the future development and innovation of oil recovery techniques. It is believed that only with bold innovations of oil recovery techniques can oil companies enhance recovery efficiency and oil quality, thereby effectively alleviating the contradiction between supply and demand of oil resources.
\end{abstract}

\section{Introduction}

With the rapid development of society and economy, people's demand for petroleum resources continues to increase, and the construction scale of the petrochemical industry is also expanding. According to the statistics of B P W orld Energy Statistical Y earbook (2019), the proven oil reserves are available for human exploitation for 50 years based on the production level in 2018. The recoverable life of Petroleum in China is 18.7 years. Global oil consumption continued to grow steadily in 2018 with a year-on-year increase of $1.5 \%$, or 1.4 million barrels per day, which is higher than the average of the past decade. Petroleum recovery is a technical means for people to obtain petroleum resources. Driven by the growing market demand, oil recovery engineering is developing at a high speed. China boasts a vast territory and abundant natural mineral resources, including petroleum. But the terrain and regional complexity and particularity bring difficulties to oil recovery, requiring more advanced oil recovery engineering techniques. The application of more advanced recovery techniques have significantly improved the quality and efficiency of oil recovery. Nowadays, the application of China's new technologies has increased the amount of oil production by nearly $33 \%$, reaching 120 million tons, under different underground oil reservoir conditions. See Figure 1-Figure 3.

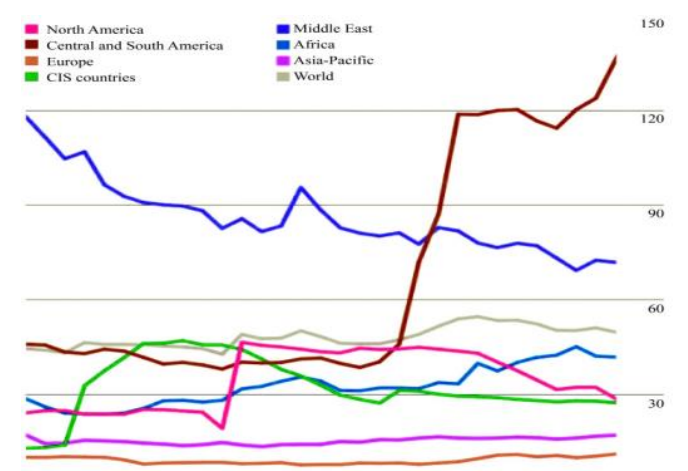

Figure 1 growth level of international oil exploitation

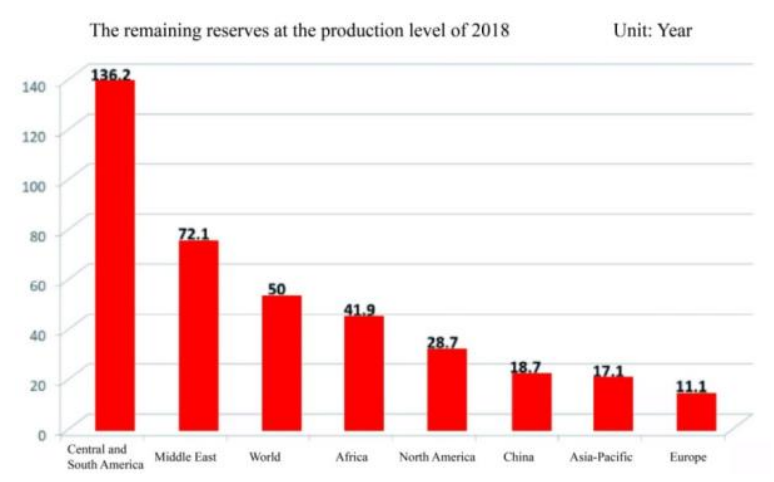

Figure 2 Statistics of oil production in international countries (2018) 


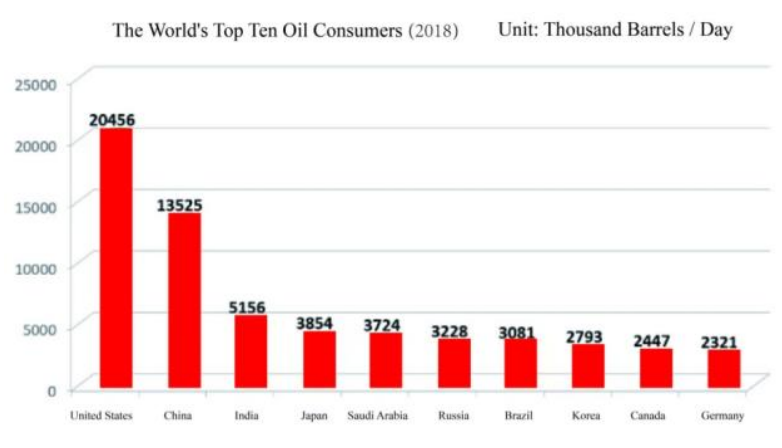

Figure 3 Statistics of the world's top ten oil consuming countries

\section{Research status}

\subsection{Status of foreign research}

Petroleum, as a kind of non-renewable energy, is renowned as the "blood of industry". Generally speaking, after primary and secondary oil recovery, $60 \%$ to $70 \%$ of crude oil, which is hard to recover, still remains in the formation. Therefore, how to improve the recovery rate of crude oil is a problem yet to be solved by oil enterprises, and it is emphasized by the oil recovery industry worldwide. Current petroleum recovery mainly adopts physical and chemical methods, such as the ASP system composed of alkaline surfactant-polymer. The global oil crisis in the 1970s sounded alarm bells for countries and made them aware of the need to accelerate research and innovation in oil recovery techniques. The 1990s has witnessed new developments of oil recovery techniques. The United States and Russia took the lead in applying microbial oil recovery technology, and is now at the stage of industrial application of the recovery techniques.

\subsection{Status of domestic research}

In China, related research on oil recovery technology started relatively late. The research on microbial technology for oil recovery began in 1966 by Xinjiang Petroleum Administration on the research of crude oil dewaxing technology. MEOR (Microbial Oil Recovery) is a scientific research project supported by the state during the Seventh Five-Year Plan period. In recent years, China has been learning the microbial oil recovery technologies from the United States and Canada, and bringing in their microbial products, which are helpful in terms of promoting the microbial oil recovery technology in China.

Yang ${ }^{[1]}$ systematically combed the three development stages of oil recovery technologies, and summarized the development of oil recovery technologies such as thermal flooding, gas flooding, chemical flooding, and microbial flooding. Wang ${ }^{[2]}$ summarized the research and application status of microbial oil recovery technologies, highlighting the difficulties in the development of microbial flooding technology. He also introduced the field test methods adopted abroad, and analyzed the future research directions of oil recovery technology and some concerned issues. Yuan and Wang ${ }^{[3]}$ systematically summarized the technologies adopted in oil fields of various properties in China, including heavy oil fields, medium-high permeability and high water-content oil fields, complex block oil fields, oilfields with special lithology, and low-permeability oil fields. He also introduced China's world-leading oil recovery technology, and studied the measures and methods suitable for various types of oil fields.

After more than ten years of development, $C$ hina's oil recovery techniques are now basically mature and can effectively handle extraction in various types of oil fields. However, it is still necessary to continuously optimize the oil recovery techniques, strengthen the understanding of recovery mechanism, and carry out technological innovation, thereby improving oil recovery efficiency.

\section{Methodology}

\subsection{Major issues in the development of oil recovery techniques}

At present, China still mainly adopts traditional oil extraction techniques for oil extraction. Some problems continue to appear during the practical application of oil recovery technology, which hinder the improvement of the extraction efficiency.

First of all, the amount of petroleum resources is limited. Therefore, with oil recovery goes on, there will be less oil resources. The oil level will get lower and the recovery rate of groundwater will be slower, which is not conducive to the large-scale pump extraction project. The temperature difference of the underground well will get larger and the oil recovery equipment in the well may be worn, which makes the electric submersible pump unable to work normally. In addition, the interaction between crude oil and rock formation will cause rusting and scaling of the oil recovery equipment, thereby shortening the service life of the equipment and reducing the oil recovery rate, which is not conducive to oil recovery.

Secondly, the temperature difference between the underground wells increases with the ongoing process of recovery, leading to poorer performance of the petroleum plugging agent in all aspects and ineffective repeated water plugging. The oil's acid resistance cannot be well controlled, resulting in the failure of effective oil recovery and loss of some oil resources. M oreover, with the continuous exploitation of petroleum, the adaptability of petroleum transportation systems to temperature differences is declining, and the normal transportation and supply of petroleum cannot be guaranteed. 


\subsection{Technical principles of oil recovery engineering in the new period}

\subsubsection{Study on the mechanism of microbial oil recovery}

The micromechanical experiment can be adopted to study the microbial oil recovery techniques. When making the simulated transparent pore model, we can take the pore groups on the cored casting slab as the object and adopt the photolithography technology. Experiment shows that the bacteria in the water phase has relatively normal morphology, and is of similar size, uniform distribution and neat arrangement. In addition, the bacteria is growing and metabolizing continuously.

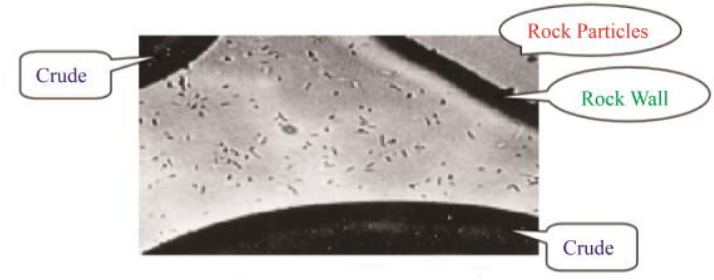

Figure 4 Distribution of bacteria in the water phase

The bacteria in the porous medium can move by themselves or with the help of injected fluid. ${ }^{[4]}$ During the secondary water flood in the water-interjected oilfields, with the help of water-injecting microorganisms, the residual crude oil adsorbed on the rock surface and was difficult to flow is emulsified and decomposed into small oil droplets or filament, which can flow again. See Figure 5.
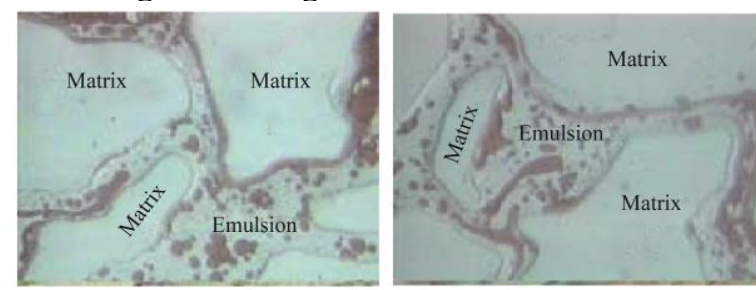

Figure 5 Form of remaining oil on the rock surface after microbial action

The whole mechanism of action can be summarized as follows:

(1) Emulsification-carry and start the remaining crude oil

The model is hydrophilic. If the pores have a highly water-drive, then water will separate the crude oil into small oil droplets, which will be surrounded by the bacterial liquid for biological and chemical interactions. The degraded oil will emulsify to different degrees, and the size of the oil droplets will also be different. These droplets will deform in the pores and then penetrate, as shown in Figure 6.
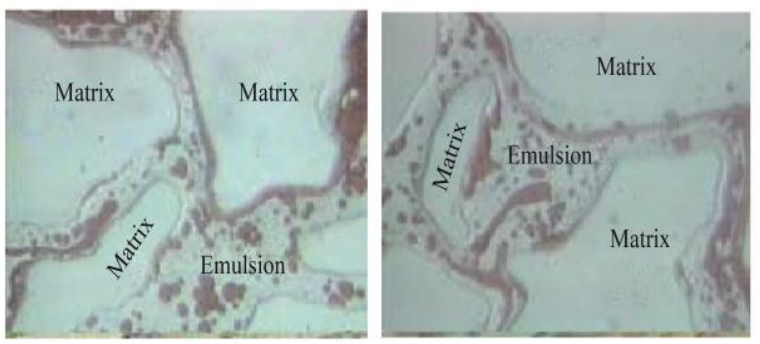

Figure 6 Emulsifying effect of microorganisms on oil

If the degree of water flooding in the pores is high, the fermentation liquid can emulsify and disperse the oil to form an emulsion, and at the same time, the oil-in-water emulsion may continue to seep.

(2) Peeling off the oil film

The wetting heterogeneous components attached to the surface of the pores interact with the heavy components in the petroleum, leaving some oil droplets in the pore walls. After the interaction of the bacteria body, the original thick oil droplets begin to be stripped under the influence of water flow, the oil film gradually becomes thinner, and the oil detaches from the oil film and become filament-like, as shown in Figure 7. The oil moves with the water and flows out of the pores of the model.
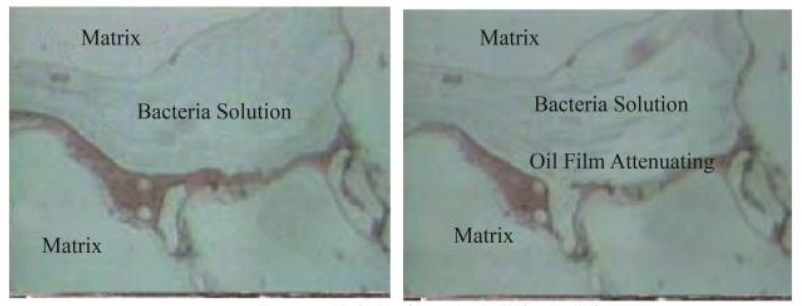

Figure 7 Detachment of residual oil after microbial action

(3) J amin effect of biogas

The Bacteria in the pores produces two kinds of biogas: mobile gas and immobile gas. See Figure 8 . The pore structure has a greater effect on the mobile gas. If the pore-throat ratio is large, the mobile gas flows slower and is easier to accumulate; on the contrary, if the pore-throat ratio is small, the mobile gas has a stronger flowing capacity. Due to the Jamin effect, immobile gas produces a certain resistance in the middle of the pores, hindering the percolation of water, and changing the direction of the fluid. Therefore, immobile gas hardly causes any resistance to the percolation of oil.
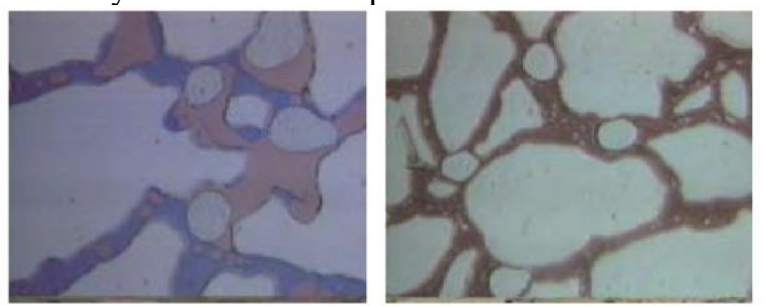

Figure 8 Biogas produced by microorganisms

\subsubsection{Principle of information technology}

Information technology has penetrated into the various 
industries, promoting the reform and development of many fields. The oil industry has also been affected by information technology. The adoption of IT in this industry has created great value for society. The application of IT in the petroleum industry is multifaceted. In the process of petroleum exploration, an information platform can be built to count and process various data in real time [5], and to systematically perform data analysis. It can be seen that information technology can be effectively connected with oil extraction.

(1) Topological structure of transmission network of information system

Oil companies at home or abroad have formed their own transmission and processing networks based on the actual situation and their own conditions. Related field data is usually transmitted to the base by means of microwave technology or satellite communication technology, which are two types of commonly used data transmission means. Figure 9 shows the network topology for data transmission using microwave technology. Figure 10 shows the network topology for data transmission using satellite communication technology.

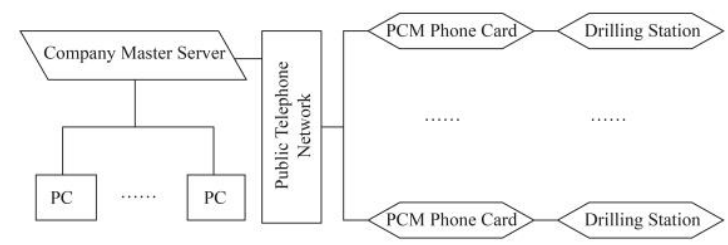

Figure 9. Data transmission network topol ogy using microwave technology

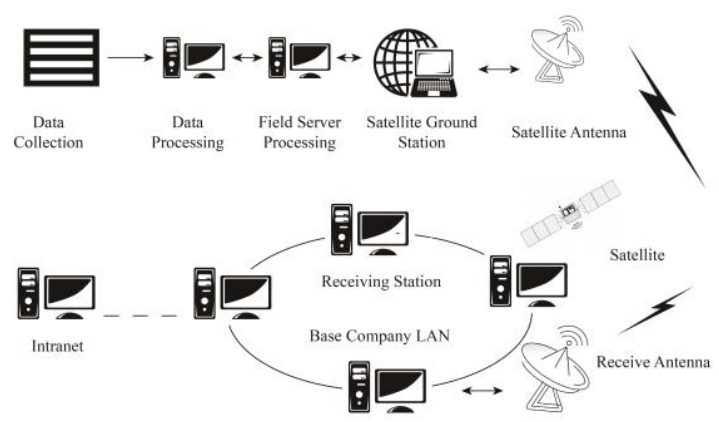

Figure 10. Data transmission network topology using satellite communication technology

(2) Data warehouse structure

Each major oilfield has certain requirements for the construction of databases and information management systems. The drilling data warehouse is mainly composed of several sub-databases, such as a drilling design database, a drilling report database, a drilling standard database, a drilling engineering database, a real-time drilling database, and a drilling history database. The drilling data warehouse of this structure can generally meet information and data demands in different links in the drilling process. Figure 11 shows the components of data warehouses and the relationships between them.

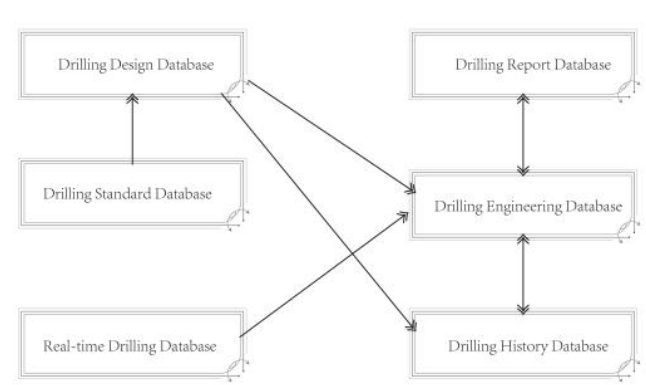

Figure 11. The basic composition and interrelationship of data warehouses

(3) Composition of information system software The software of the information system can cover the most basic summarized data and more complicated intelligent design. Its composition is shown in Figure 12.

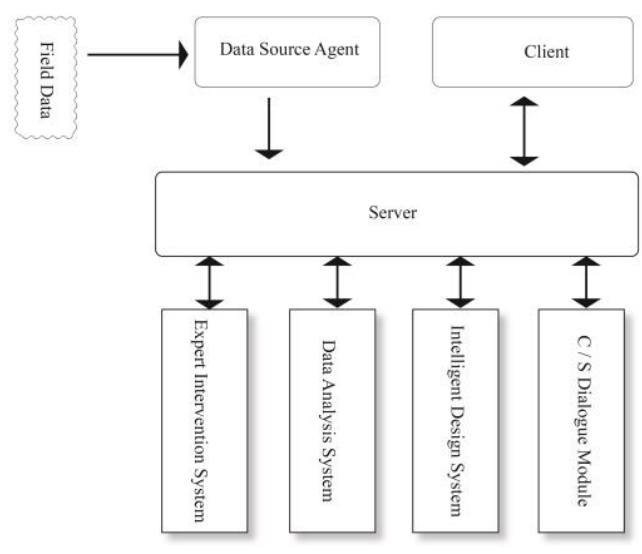

Figure 12. Schematic diagram of the structure of information system software

The client-side in the information system software has multiple functions and is composed of multiple modules, including real drilling parameter arrangement, trajectory design, $\mathrm{C} / \mathrm{S}$ dialogue, target prediction, mechanical analysis, geomagnetic calculation, and final well report. Server-side software has most of the above-mentioned client software modules as well as the functions of management and analysis.

\section{Results and discussion}

\subsection{Innovative application of microbial technology and information technology}

\subsubsection{Innovative applications of microbial technology}

If the connecting layer develops well in the direction of both water wells and oil wells, or only in the direction of oil wells, and if the water content of the oil field is below $80 \%(60 \%$ to $80 \%$ for the best), then we can use microbial flooding. If the oil well is relatively thick with lower fluid production and a water content of $15 \%$ to $60 \%$, and if it has a relatively large potential for tapping, and at the same time, if the daily fluid production is initially high but then decreases rapidly, then we can use microbial stimulation. If the well develops well only in 
the direction of water well and the water content is less than $60 \%$, then we can adopt microbial fracturing.

In terms of economic benefits, microbial fracturing comes first, followed by microbial flooding and microbial huffing-puffing. However, the gap between microbial flooding and microbial huffing-puffing is very small, with little difference in total input and output ratios. However, from the aspect of construction, microbial fracturing comes first. Microbial fracturing is performed at the same time as fracturing construction, and requires no additional workload and construction investment during the fracturing construction process. So it can deliver the best benefits. For a non-fractured well, microbial flooding can be more effective than microbial huffing-puffing, which is because microbial flooding can inject microorganisms from the water injection well, and can be taken out without the need of closing the well, and is wider in terms of the control range. Microbial huffing-puffing is convenient and economical for blocked wells around wellbores.

In summary, microbial oil recovery techniques can promote the oil recovery efficiency of low-permeability oilfields. They are desirable techniques with bright application prospects.

\subsubsection{Innovative application of information technology}

(1) With the help of communication network technology, we can dynamically reproduce various logging information and data so as to more scientific decisions. We can also promote the standardized development of the drilling industry and equip oil companies with modern science and technologies.

(2) With the help of remote data transmission in information technology, the base experts can monitor the site in real-time, and identify, analyze, and evaluate the complex problems encountered in drilling operations online. In this way, they can propose feasible solutions for subsequent work, eliminate hidden risks, and ensure scientific and effective decision-making.

(3) As the software system is continuously upgraded and expanded with greater information processing capabilities, network and data resources can be fully and effectively utilized. The intelligent software can be used to adjust and optimize the drilling construction plan in real time, and to ensure the scientificity and accuracy of the construction plan through trajectory control and performance monitoring of the drilling fluid. It is necessary to achieve drill efficiency and accuracy, and at the same time, protect the environment and gasoline layers.

(4) The data warehouse can enhance the degree of data sharing by simultaneously collecting data from multiple platforms and updating in real-time, thereby greatly improving the efficiency of oil extraction and management. Through video conferencing, we can adjust the operation plan according to the on-site operation conditions, thereby realizing cross-border monitoring of drilling operations. With a more comprehensive data service system, we are capable of monitoring the entire operation of oil extraction, and more accurately control the drilling and extraction process.

\subsection{Innovative applications of other techniques}

\subsubsection{Innovative applications of new materials}

Various new materials will be used in the process of oil extraction. The most commonly used new materials are polymer materials ${ }^{[6]}$, which indicates that the oil recovery techniques have entered a modern development stage to a certain extent. During oil extraction, we can also use some materials with higher abrasion resistance, which can improve not only the mining intensity, but also the survey level. Materials with strong anticorrosive properties or with protective effects can be used to better protect the equipment, improve oil recovery rate, and improve the overall quality of the entire project. Therefore, new materials can help to promote the innovation in oil recovery techniques. See Figure 13.

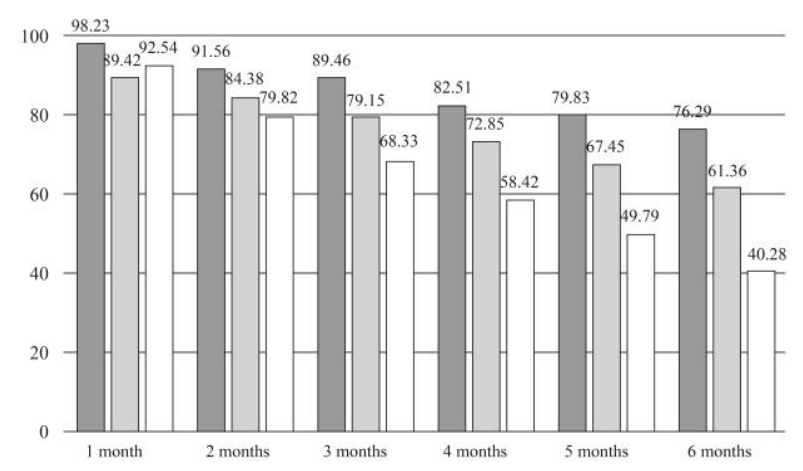

Figure 13. Growth rate of oil recovery technique effect in different periods with the application of new materials

\subsubsection{Technical innovation of thick oil layers}

Thick oil layer technique, as a key technique in oil extraction, can integrate related oil production techniques to ensure the quality of oil production as a whole. The successful implementation of this technique requires the use of formulation as the auxiliary agent, which mainly plays a role in reducing the gelation time. The formulation should be of high performance, high economic advantages, and low cost. Thick oil layer technique is conducive to improving the utilization rate of important materials in oil recovery, thereby providing a guarantee for the scientific and standardized production.

\subsubsection{Technical innovation of horizontal wells}

Different oil mines are located in various environments with different geological features and great complication. Therefore, before implementing the oil recovery project, it is necessary to analyze the geological environment of the mining site. Fracturing technique is based on the analysis of the geological environment, and different environments require different fracturing techniques. Detailed information around the well, especially at the 
bottom of the well, should be collected as the basis for the fracturing technique. The fracturing mode shall be matched with horizontal wells to ensure the scale of the oil recovery project. In addition, we shall al ways pursue technological innovation, continuously develop new oil recovery techniques, and optimize the original ones, thereby continuously improving oil recovery rate and meeting various needs. See Figure 14.

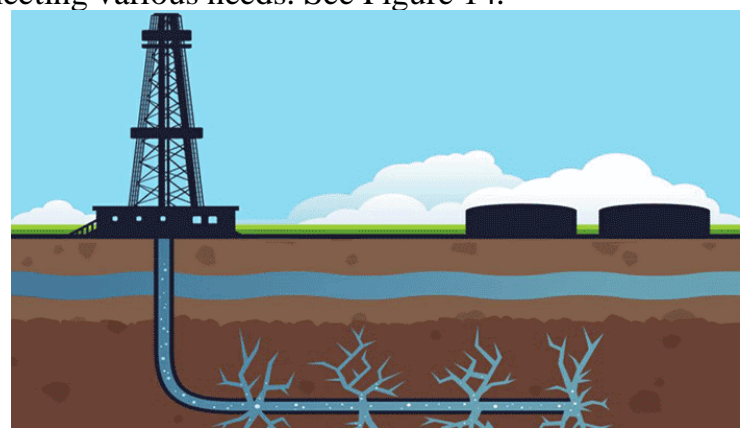

Figure 14. Horizontal fracturing and horizontal well technique

\subsection{Measures to promote technological innovation in oil extraction}

Petroleum provides the material foundation and energy support for the development of the national economy. In the new period, there has been tremendous progress in oil extraction technology, which greatly promotes the oil recovery rate, thereby ensuring the development of the national economy, facilitating people's lives, and improving the quality of life. However, as we all know, petroleum is a non-renewable resource with a limited amount. In order to realize the reasonable exploitation of petroleum resources and promote the sustainable development of China's economy, we must continuously research and develop new oil recovery techniques and improve the original oil recovery methods so as to increase oil recovery rate, and standardize management and supervision of the oil recovery process.

First, it is necessary for oil exploration companies to strengthen their understanding and recognition of sustainable development, and to take sustainable development as the core when formulating corporate development strategies to continuously improve their competitiveness. Secondly, it is necessary to increase investment in research and development of new oil extraction technologies, strengthen the intensity of oil extraction, and improve the efficiency of oil extraction. Third, it is necessary to promote the concept of a thrifty and frugal life, formulate relevant standards for the daily oil consumption of the government and residents. The standards shall be strictly enforced, thereby reducing the waste of resources and increasing utilization rate.

\section{Conclusion}

The 21st century witnesses the rapid development of various high-tech industries, including Internet information technology, nano-new materials, biological industries, and energy industries. New developments in petroleum extraction engineering techniques has greatly improved the efficiency of oil extraction. But we still face many difficulties in oil recovery, which need to be handled by oil companies through the development of new technologies and methods.

The exploitation of petroleum resources is featured with complexity and technical demands. It involves the application of technical and scientific knowledge, and also integrates many disciplines. The level of technological application directly affects the extraction efficiency and petroleum quality. As one of the major industries in China's national economic development, the petroleum industry plays a very important role in social and economic development in our country. Therefore, continuous innovation and development of oil recovery techniques are of practical significance, and are regarded as the biggest driving force for the sustainable development of the petroleum industry.

\section{References}

1. Zhigang $Y$ ang, tertiary oil recovery technology and progress, chemical progress, 30 (S1), pp.420-423, (2011).

2. Weidong Wang, microbial oil recovery technology research and test, oil drilling and production technology, 34 (01), pp.107-113, (2012).

3. Shiyi Y uan, Qiang W ang, new progress and Prospect of main technologies for oilfield development in China, petroleum exploration and development, 45 (4), pp.12, (2018).

4. Fangling Qin, Yue fan, Haitao Bai, Research progress of microbial oil recovery technology, Modern chemical industry, v.36; no.351 (01), pp.53-56 + 58, (2016).

5. J unping Wang, A pplication and Prospect of polymer materials in oil production engineering, Chemical management, 000 (015), pp.113, (2014).

6. W ei Li, Chunyu Zhao, M anagement and application of "big data" in oilfield exploration and developmen, Information technology, (4), pp 196-198, (2013). 